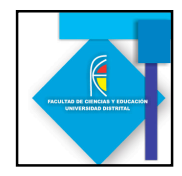

\title{
REFLEXIONES SOBRE EL TIEMPO EN LA FÍSICA
}

\section{REFLECTIONS ON TIME IN PHYSICS}

\author{
Juan Carlos Castillo Ayala ${ }^{1}$
}

\section{Resumen}

El presente trabajo contiene algunas reflexiones en torno a la idea de tiempo y cómo se asume su carácter fenomenológico en algunas teorías científicas. Por último, se hacen algunas reflexiones sobre la complejidad que reviste la pregunta por el tiempo.

Palabras clave: Tiempo, temporalidad.

\section{Abstract}

This paper contains some reflections on the idea of time and how it assumes its phenomenological character in some scientific theories. Finally we make some reflections on the complexity of the question time.

Keywords. Time, temporality

\section{Introducción}

Qué es el tiempo... esta es una cuestión aparentemente simple, ya que para todos, el tiempo está implícito en la comprensión de nuestra vida y de los hechos de la naturaleza. Pero esta cuestión toma una dimensión más amplia cuando nos preguntamos por aspectos tales como: ¿Qué experiencia tenemos del tiempo?, es decir qué elementos nos permiten organizar las experiencias de acuerdo a una temporalidad, ¿existe en nosotros alguna conciencia del tiempo?, ¿Acaso es el tiempo una condición del conocimiento humano? ¿Es posible afirmar que, si existe alguna, la experiencia y la

conciencia que tenemos del tiempo es común a todos?, ¿ siempre experimentamos el tiempo de la misma forma, con el mismo ritmo y la misma cadencia, en las diferentes situaciones y etapas de nuestra vida? Estas son algunas preguntas que nos podemos

\footnotetext{
${ }^{1}$ Profesor Departamento de Física. Universidad Pedagógica Nacional, jccastillo@pedagogica.edu.co
} 
$5^{\circ}$ CONGRESO NACIONAL DE ENSEÑANZA DE LA FÍSICA.

Universidad Pedagógica Nacional (U. P. N)

Universidad Distrital Francisco José de Caldas (U. D. F. J. C)

Bogotá, Colombia. 16 al 20 de mayo 2011

hacer en relación con el tiempo, mas aun, pueden haber muchas más

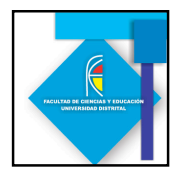

preguntas, y por supuesto de mayor profundidad, pero éstas se plantean a manera de ilustración de la complejidad de la cuestión en relación con el tiempo.

Ahora bien, para ilustrar un poco más la complejidad que reviste la pregunta por el tiempo, se presenta una breve reseña de algunas de las concepciones acerca del tiempo, que se pueden evidenciar en las ciencias naturales, haciendo un análisis desde algunos aspectos de orden filosófico.

\section{El Tiempo en las Teorías Científicas}

\section{El tiempo en la Mecánica}

El tiempo forma parte estructurante de las teorías científicas, especialmente en la mecánica clásica y la perspectiva mecanicista es evidente su papel determinante, ya que en las descripciones y explicaciones se involucra el tiempo como un elemento fundamental, aspecto que se pone de manifiesto en el hecho de que las descripciones se dan en términos de ecuaciones diferenciales con respecto al tiempo. Ahora bien, uno de los aspectos que puede resultar problemático, en relación con idea intuitiva de tiempo, es la manera como éste se involucra en tales descripciones, puesto que no establece distinción alguna entre pasado y futuro, ya que las ecuaciones están planteadas de una forma simétrica con respecto al tiempo; aspecto que implica que la descripción de un evento físico no distinga la evolución del mismo, esto es, no hay diferenciación entre describir hacia el pasado y hacia el futuro. Este elemento pareciera ser una imprecisión formal, por llamarla de alguna manera, pero, es claro que la idea de tiempo absoluto homogéneo y lineal que está a la base de la mecánica de corte newtoniano no posibilita establecer una diferencia entre el pasado y el futuro, ya que la homogeneidad implica que no se pueda hacer distinción entre un instante y otro; así en el ámbito de la mecánica la distinción entre pasado y futuro se involucra como un elemento puramente "fenomenológico" que no forma parte estructural de la teoría misma.

En este sentido se puede afirmar que la asimetría temporal, no contemplada en la teoría de la mecánica clásica, está estrechamente relacionada con la experiencia y con la conciencia del tiempo, siendo desde aquí que se puede pensar un tiempo regido por una direccionalidad que se puede expresar en los siguientes términos.

"pienso naturalmente en leyes de índole tan consabida como las siguientes: que el orden temporal fijo es una serie bidimensional; que dos tiempos diferentes nunca

pueden ser simultáneos; que su relación no es simétrica; que en el tiempo rige la transitividad; que todo tiempo tiene un tiempo anterior y posterior, etc. ${ }^{13}$

\footnotetext{
${ }^{2}$ Fenomenológico en este caso hace referencia a un aspecto que forma parte de nuestra experiencia y conciencia, pero que no está contemplado en la teoría, ni en su formalización. Más adelante se abordará con más precisión el término

${ }^{3}$ HUSSERL, E. Lecciones de Fenomenología de la Conciencia interna del tiempo. Editorial Trotta. Madrid.
} 
$5^{\circ}$ CONGRESO NACIONAL DE ENSEÑANZA DE LA FÍSICA.

Universidad Pedagógica Nacional (U. P. N)

Universidad Distrital Francisco José de Caldas (U. D. F. J. C)

Bogotá, Colombia. 16 al 20 de mayo 2011

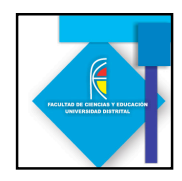

Ahora bien, ver algunos otros elementos de la concepción de tiempo presente en la mecánica de corte newtoniano y en la perspectiva mecanicista. La categoría de tiempo homogéneo e isotrópico de la mecánica newtoniana es la que se le atribuye al tiempo absoluto, el cual tiene existencia ontológica, en el sentido que para Newton, el tiempo existe independientemente de la experiencia que se tenga del mismo, aspecto que expresa de la siguiente manera.

El tiempo absoluto, verdadero y matemático*, en sí y por su propia naturaleza sin relación a nada externo fluye uniformemente y se dice con otro nombre duración.

Además del tiempo absoluto Newton define el tiempo relativo, el cual está relacionado con la medida de la duración, este tiempo es del cual se tiene experiencia sensible y del cual se puede tener una conciencia, en el sentido que está en íntima relación con los eventos, con la sucesión y repetición de los mismos, ya que la medida está relacionada con el movimiento que es sensible, así el movimiento se puede considerar como la forma de sensibilidad del tiempo.

El tiempo relativo aparente y vulgar es alguna medida sensible y exterior(precisa $o$ desigual) de la duración mediante el movimiento, usada por el vulgo, en lugar del tiempo verdadero, día, mes, año son medidas semejantes.

Fijémonos como en la concepción newtoniana del tiempo están presentes dos elementos, uno es el tiempo como tal, el tiempo en si mismo, y la manifestación del mismo, así se podría decir que el tiempo absoluto de Newton se sitúa como un tiempo objetivo, y como tiempo objetivo, no se tienen datos fenomenológicos del mismo; en tanto que el tiempo relativo aparente y vulgar puede ser situado como el tiempo que deviene de la posibilidad de una conciencia del mismo, del tiempo que aparece en términos de una duración que aparece, cuya aparición está relacionada con el movimiento, el movimiento como un evento temporal, en el sentido de que éste se sitúa como una vivencia la cual es posible organizar en términos de momentos, momentos que son la base para la experiencia de lo temporal, que de ningún modo se puede decir que sean el tiempo absoluto-el tiempo objetivo-sino, que se constituyen en datos fenomenológicos del tiempo.

En este contexto de reflexión es posible preguntarse sobre la relación que se puede establecer entre el tiempo absoluto-situado como tiempo objetivo- y el tiempo relativosituado como dato fenomenológico. Es posible hacer una interpretación en la cual el tiempo relativo está relacionado con el tiempo absoluto en el sentido que el primero es un dato fenomenológico que por medio de una cierta función aprehensiva expone al segundo, es decir, el tiempo relativo no es el tiempo absoluto, sino una manifestación

de éste, manifestación que expone la duración como cualidad del tiempo absoluto, el tiempo relativo manifiesta la duración del tiempo absoluto, ya que la experiencia del tiempo relativo se da a través del movimiento como objeto temporal, y la percepción

\footnotetext{
${ }^{*}$ Matemático en este contexto se refiere a lo perfecto, regido por reglas perfectas provenientes de una conciencia perfecta.
} 
$5^{\circ}$ CONGRESO NACIONAL DE ENSEÑANZA DE LA FÍSICA.

Universidad Pedagógica Nacional (U. P. N)

Universidad Distrital Francisco José de Caldas (U. D. F. J. C)

Bogotá, Colombia. 16 al 20 de mayo 2011

del objeto temporal tiene en sí misma temporalidad-la duración del

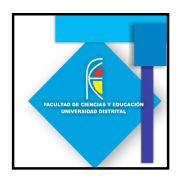

movimiento presupone la duración misma de la percepción del movimiento-.

\section{El Tiempo en la Teoría de la Relatividad}

La teoría de la relatividad desecha la idea de tiempo absoluto verdadero y matemático $^{* *}$, asumiendo una idea de tiempo como relación entre eventos, estoes, el tiempo da cuenta de la organización de los eventos, ya sea mediante una sucesión, en la cual unos eventos anteceden a otros, unos eventos suceden a los otros, o hay eventos que son simultáneos. Particularmente para esta organización la idea de simultaneidad es fundamental, ya que la organización temporal de los eventos físicos deviene de la simultaneidad-si digo que el tren llego a la estación a las cinco, lo que estoy afirmando es que la llegada del tren a la estación se dio simultáneamente (coincidió) con el evento de que las manecillas del reloj, de la estación, marcaban las cinco; aun más, si digo que la llegada del tren a la estación fue simultánea a la llegada de otro tren a una estación distante, esto es ambos trenes llegaron a su estación respectiva a las cinco, lo que estoy afirmando es que ambos trenes llegaron a su estación simultáneamente al evento de que las manecillas del reloj, colocado en cada estación, marcarán las cinco, bajo la condición que juntos relojes estuviesen sincronizados ${ }^{4}$.

Ahora bien, en este sentido un evento antecede al otro si la marcación del reloj simultánea con tal evento antecede a la marcación simultánea con el otro, bajo la condición de que los relojes estén sincronizados. Otro aspecto importante es que eventos simultáneos vistos desde un cierto marco de referencia pueden no serlo vistos desde otro marco de referencia-si simultáneamente a la llegada de los trenes a sus respectivas estaciones se envía una señal luminosa de una estación a la otra y de la segunda a la primera, un observador que se encuentre en reposo a la mitad de la distancia entre las estaciones recibirá las dos señales simultáneamente, con lo cual deducirá que la llegada de los trenes a las estaciones respectivas fue simultánea; si otro observador se mueve de la primera estación a la segunda, pasa por la mitad de la distancia entre las estaciones simultáneamente al evento que el primero recibe las señales, este segundo observador recibirá primero la señal proveniente de la segunda estación que la proveniente de la primera, con lo cual concluirá que la llegada del tren a la segunda estación antecede a la llegada del otro tren a la primera estación, esto es la recepción de la señal proveniente de la segunda estación, para este observador, coincide con una marcación de las manecillas de su reloj que antecede a la marcación que coincide con la recepción de la segunda señal.

Habiendo hecho una descripción sucinta de la manera como se concibe el tiempo en la teoría de la relatividad, fijémonos como esta teoría no establece ni indaga sobre la existencia de un tiempo objetivo, sino simplemente asocia el tiempo a una organización

\footnotetext{
${ }^{* *}$ Matemático en el sentido Newtoniano

${ }^{4}$ La sincronización de relojes distantes es un procedimiento que se hace tomando en cuenta el intervalo de tiempo que demora en viajar una señal luminosa de un lugar al otro, es decir es un procedimiento que no se hace de manera directa.
} 
$5^{\circ}$ CONGRESO NACIONAL DE ENSEÑANZA DE LA FÍSICA.

Universidad Pedagógica Nacional (U. P. N)

Universidad Distrital Francisco José de Caldas (U. D. F. J. C)

Bogotá, Colombia. 16 al 20 de mayo 2011

de los eventos físicos, organización que no es manifestación de

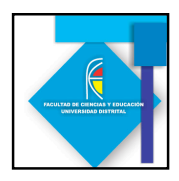

naturaleza alguna de un tiempo objetivo, sino que es en sí misma el tiempo; en este orden de ideas, el tiempo de la relatividad es un tiempo fenomenológico, en el sentido que deviene de la experiencia y de la conciencia del individuo, experiencia y conciencia de la simultaneidad o sucesión de eventos, así el tiempo de la relatividad está anclado en la conciencia de los eventos físicos, conciencia que lleva a hacer una organización temporal de los mismos.

\section{El Tiempo de la Evolución}

Aquí se abordará la manera como puede ser entendido el tiempo desde las teorías que se refieren a la evolución, para tal propósito se mostrarán algunos aspectos de dos teorías científicas que hacen referencia a la evolución, estas son la teoría de la evolución de Darwin y la termodinámica, teoría que explora la evolución de los sistemas a partir de el concepto de proceso, particularmente, de proceso irreversible.

La teoría de la evolución de Darwin basada en la idea de adaptación se aleja de las ideas- provenientes de la tradición judeocristiana-que asumen que en las especies las formas y funciones de sus diversos órganos proceden de un "diseño", por tomar algún ejemplo, el pico de las águilas tiene forma de gancho, ya que está diseñado para rasgar la carne de sus presas; la teoría de la evolución asume que la forma función que tienen los diversos órganos de las especies proviene de un proceso de adaptación a las diversa condiciones de supervivencia por las que deben atravesar. Así pues, la forma y la función están ligadas con un proceso, proceso que imprime un carácter histórico a las especies, esto es en las formas y funciones actuales está el rastro de las condiciones pasadas, y hace prever que las formas y funciones futuras estarán impresas con las condiciones actuales.

Fijémonos como asumir las formas y funciones ligadas a un proceso de adaptación las sitúa como datos temporales, es decir que la evolución es un evento temporal, evento temporal que presupone una duración, duración de los estados, referidos a las formas y funciones, anteriores y la duración de los estados actuales, como también la duración del cambio mismo, ya que un estado no puede ser otro, el cambio de un estado al otro presupone una duración; en este orden de ideas, la mutación que sufre una especie por adaptación está ligada a una duración. Por otra parte, la idea de evolución, de evolución adaptativa, imprime una asimetría temporal entre pasado y futuro, ya que incluye la historia como parte fundamental de la explicación, entendiéndose aquí la historia como una forma de temporalidad que distingue el pasado del futuro, la historia es asumida como forma de temporalidad, ya que la historia es en sí un objeto temporal que hace conciencia sobre un devenir de cosas, de estados, de eventos, que se organizan de acuerdo con un orden, orden que presupone la duración de un trecho entre la estado y otro; duración que aparece cuando la historia se hace relato.

Notemos que la temporalidad que aparece deviene de la asunción del proceso que se ancla en las evidencias de pasados distantes, pasados distantes que se pueden 
$5^{\circ}$ CONGRESO NACIONAL DE ENSEÑANZA DE LA FÍSICA.

Universidad Pedagógica Nacional (U. P. N)

Universidad Distrital Francisco José de Caldas (U. D. F. J. C)

Bogotá, Colombia. 16 al 20 de mayo 2011

se

transformar en relatos ${ }^{5}$, relatos que son objetos temporales; si bien no

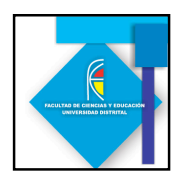

experimenta la duración del proceso, se tiene experiencia de la duración de la historia a través de los relatos construidos con las evidencias de los pasados distantes, aquí los datos fenomenológicos desde los que se constituye la conciencia del tiempo de la evolución, no son otra cosa que los relatos construidos con evidencias de pasados distantes.

Por su parte la termodinámica se establece como la teoría de los procesos, el proceso supone un cambio que tiene una duración determinada, es decir tiene un principio y tiene un fin, esto es, parte de un estado y llega a otro, poniéndose de manifiesto que el cambio requiere una duración, dígase por ejemplo, una tasa de café caliente dejada sobre la mesa se enfría hasta llegar a la temperatura ambiente, este cambio aparece como un objeto temporal, en el sentido de que la tasa de café no puede en un mismo instante estar caliente y fría, sino que este cambio tiene una duración, duración que se percibe- la percepción de la duración en si misma tiene duración-y que no solamente es una experiencia temporal, sino que además es la experiencia misma del procesotenemos la experiencia del café caliente y del café frío, pero no del proceso de enfriamiento- es la duración la que nos provee la experiencia del proceso, el proceso como objeto temporal aparece a través del cambio.

Nótese un elemento que llama la atención, en primera instancia el proceso puede ser entendido como un dato fenomenológico del tiempo, en el sentido que el proceso es un objeto temporal, pero no es el proceso el dato fenomenológico, sino la duración del cambio es la que instaura al proceso como objeto temporal, y asimismo es la duración el cambio el dato fenomenológico del tiempo.

Por otra parte, las restricciones a los procesos que se ponen de manifiesto con la segunda ley de la termodinámica establecen plenamente una asimetría temporal entre pasado y futro: "La dirección del tiempo está gobernada por el crecimiento de la entropía", esto es los procesos son la evolución misma, puesto que dan en una determinada dirección, la tasa de café caliente se enfría espontáneamente hasta llegar a la temperatura ambiente, pero la tasa de café no se caliente espontáneamente desde la temperatura ambiente hasta llegar a estar caliente nuevamente, de la misma manera que un viejo no se hace joven; aspectos que muestran que los procesos son irreversibles, irreversibilidad que es manifestación de la simetría temporal entre pasado y futuro, es decir es manifestación de la dirección del tiempo.

Por otra parte, la segunda ley de la termodinámica también puede entenderse como la que da cuenta de las condiciones de posibilidad de que se den los procesos, así la condición de posibilidad de un proceso es la manifestación de la cualidad creadora del tiempo.

\footnotetext{
${ }^{5}$ Relato se asume como cronologías, mapas temporales, relatos en el sentido habitual, en general formas de manifestar el carácter histórico.
} 


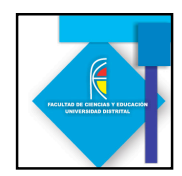

\section{Algunas Consideraciones Finales}

Las anteriores formas de entender el tiempo pueden ser consideradas como una síntesis gruesa de las concepciones de tiempo que están a la base de la ciencia moderna; aspecto que pone de manifiesto, que aunque la ciencia moderna está fuertemente anclada en lo que se conoce como cultura occidental, la diversidad de concepciones de tiempo que se encuentran a la base de las diferentes teorías son una clara muestra de que no hay un único tiempo, más aun, si pensamos en heterogeneidad cultural de un país como el nuestro es claro que también hay una heterogeneidad de maneras de concebir el tiempo y de relacionarse con su devenir.

Por otra parte, esta diversidad de tiempos y de formas de experiencia temporal, son una muestra, muy pequeña, pero clara de la complejidad que reviste la pregunta por el tiempo, ya sea que se haga desde las ciencias naturales, como desde la filosofía o desde la antropología y las ciencias sociales y humanas, complejidad que muestra una ruta muy amplia de trabajo e investigación.

El intento de análisis fenomenológico de los diversos tiempos que están en algunas teorías científicas se plantea como un reto para hacer un análisis mucho más profundo que devele algunos otros aspectos, tanto de los diversos tiempos de I ciencia, como de la fenomenología del tiempo.

Este primer análisis pone de manifiesto que la complejidad de la pregunta por el tiempo no puede dejarse de lado en la enseñanza de las ciencias, puesto que le tiempo en las ciencias no es único, aspecto que hace necesario establecer escenarios de reflexión sobre los diversos tiempos y temporalidades, más aun si consideramos que hay también diversos tiempos ligados a los espacios de significación de los individuos y los grupos humanos.

\section{Referencias Bibliográficas}

Ayala, M. M, MALAGÓN, F. CASTILLO, J. C. GARZÓN, M. (2003). La elasticidad y la perspectiva de estados. VIII Conferencia Interamericana sobre Educación en la Física. Habana: Universidad de la Habana.

Ayala, M. Malagon, F. Castillo, J. C. Garzón , M. (2003). La mecánica y su relación con otras teorías. Elementos para un análisis de alternativas para la enseñanza de la mecánica. Anales VIII Conferencia Interamericana sobre Educación en Física . La Habana Cuba.

Baillon, J. F. (2007). Teología newtoniana y teoría de la visión: qué contextos para las ediciones de la óptica de Newton de 1704 a 1722. (U. d. Antioquia, Ed.) Estudios Filosóficos (35), 55-66.

Bernardini, C. Tarsitani, M. (1998). Thinking Physics for teaching. Thinking Physics for teaching . 


\section{$5^{\circ}$ CONGRESO NACIONAL DE ENSEÑNANZA DE LA FÍSICA. \\ Universidad Pedagógica Nacional (U.P.N) \\ Universidad Distrital Francisco José de Caldas (U. D. F. J. C) \\ Bogotá, Colombia. 16 al 20 de mayo 2011}

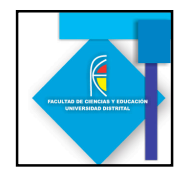

Cabo, J. \& Enrique, C. (2004). Hacia un Concepto de Ciencia Intercultural. Enseñanza de las ciencias , 22 (1), 137-146.

Castillo, J. C. (2004). El concepto de corriente y la perspectiva dinámica. Universidad Pedagógica Nacional. Facultad de Ciencia y Tecnología. Departamento de física, Bogotá.

Claret, A. (2003). Cuestiones Históricas y Epistemológicas en torno a la Enseñanza de las Ciencias. En Educación y Formación del Pensamiento Científico. Cali: Instituto de Educación y Pedagogía Universidad del Valle.

Cobern, W. \& Loving, C. (2001). Defining "Science" in a multicultural world: Implications for Science education. Science Education , 85, 50-67.

Cobern, W. (1996). Worldview theory and conceptual change in science education. Science Education , 80 (5), 579-610.

Cohen, B. (1983). La revolución newtoniana y la transformación de las ideas científicas. Madrid, España: Alianza.

Eistein, A. (1922). Sobre la toría especial y la teoría genral de la relatividad El significado de Relatividad. Planeta Agostini.

Elkana, Y. (1983). La ciencia como sistema cultural: Una aproximación antropológica. Boletin sociedad Colombiana de Epistemología , 65-80.

Ferrer, A. (2004). Concepcoes de estudantes acerca do conceito do tempo: uma análise á luz da epistemología de Gastón Bachelard. (U. d. Educacao, Ed.) Sao Paulo, Brasil.

Fried Schnitman, D. (1995). Nuevos paradigmas, Cultura y subjetividad. Barcelona: Paidos.

Granes, J. \& Caicedo, L. (1997). Del contexto de producción de conocimientos al contexto de la enseñanza. Análisis de una experiencia pedagógica. Revista Colombiana de Educación (34), 72-73.

Grupo Física y Cultura. (2000). Grup Lac. Obtenido de www.colciencias.gov.com.

Gutierrez, C. (2006). La Flecha del tiempo. Ciencia ergo sum , 13 (3), 246-252.

Hertz, H. (1899). Los principios de la mecánica.

Hiedegger, M. (1927). El ser y el tiempo. Fondo de cultura económica.

Husserl, E. (1928). Lecciones de fenomenología de la conciencia interna del tiempo. Trotta.

Kunh, T. (1970). La Estructura de las Revoluciones Científicas. Mexico: Fondo de Cultura Económica. 


\section{$5^{\circ}$ CONGRESO NACIONAL DE ENSEÑNANZA DE LA FÍSICA. \\ Universidad Pedagógica Nacional (U.P.N) \\ Universidad Distrital Francisco José de Caldas (U. D. F. J. C) \\ Bogotá, Colombia. 16 al 20 de mayo 2011}

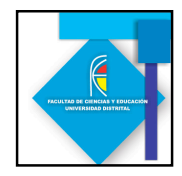

Leibnitz, G. en L. H. Leomker (ed). (1956). Philosophical Papers and Letters.

Mathews, M. (1994). Historia, Filosofía y Enseñanza de las Ciencias: La aproximación actual. Enseñanza de Las Ciencias , 12 (2), 255-277.

Minkowski, H. (1908). Discurso inaugural de la $80^{\circ}$ Reunión de la Asamblea general Alemana de científicos naturales y físicos.

Molina, A \& Mojica, L. (2004). Las teorías de la evolución en los textos escolares: análisis crítico histórico-epistemológico de Philipp Mathy. Cuadernos de investigación (4), 69-91.

Molina, A. (2002). Conglomerado de relevancias y formación científica de niños, niñas y jóvenes. Revista científica, 187-199.

Molina, A. Sepúlveda, C. López, D. Mojica, L. Espitia, M. (2004). Las fuentes de conocimiento, selección de contenidos y textos didácticos. Enfoques culturales en la educación en ciencias el caso de la evolución de la vida. Cuadernos de investigación. , 2633.

Moreira, M. (2005). Una visión toulminiana respecto a la disciplina investigación básica en educación en ciencias: el rol del foro institucional. Ciencia \& Educasao , 11 (2), 181-190.

Newton, I. (1687). Principios matemáticos de la filosofía natural. Barcelona, España: Barcelona.

Olaya, S. (2007). Memorias IV Coloquio latinoamericano de fenomenología " Fundadores de la Fenomenología". Bogotá.

Ortiz, R. (2007). Anotações sobre o universal e a diversidade. Revista Brasileira de Educação, 12 (34).

Pedreros, R. Castillo, J. C. (2007). La organización de los Fenómenos térmicos. Modulo de trabajo, Universidad Pedagógica Nacional. Facultad de ciencia y Tecnología. Departamento de Fisica, Bogotá.

Pinto, A. (2004). Concepcoes de estudantes acerca do conceito de tempo:uma análise a luz da epistemología de Gastón Bachelard. En U. d. Educacao (Ed.).

Plank, M. (1945). Treatise on Termodynamics . New York, USA: Dover.

Pozo, J. Gomez, M. (1998). Aprender y enseñar ciencia Del conocimiento cotidiano al conocimiento cietífico. Madrid: Morata.

Prigogine, I. (2001). El fin de las certidumbres. España: Taurus.

Prigogine, I. (1999). Las leyes del caos. Barcelona: Crítica. 


\section{$5^{\circ}$ CONGRESO NACIONAL DE ENSEÑANZA DE LA FÍSICA. \\ Universidad Pedagógica Nacional (U.P.N) \\ Universidad Distrital Francisco José de Caldas (U. D. F. J. C) \\ Bogotá, Colombia. 16 al 20 de mayo 2011}

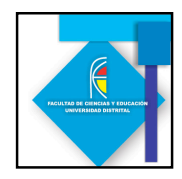

Reichenbach, H. (1958). The Philosophy of Space an Time. New York:

Dover.

Ricoeur, P. Larre, C. Panikkar, . Kagame, A. Lloyd, G. Neher, A. Pattaro,G. Gardet, L.Gouveritch, A. (1975). Las culturas y el tiempo. Estudios resumidos para UNESCO. Vozes. Editorila de la Universidad de Sao Paulo.

Ricoeur, P. Larre, C. Panikkar, . Kagame, A. Lloyd, G. Neher, A. Pattaro,G. Gardet, L.Gouveritch, A. (1975). Las Culturas y el Tiempo. Estudios Resumidos para UNESCO. Sao Paulo: Vozes. Editorial de la Universidad de sao Paulo.

Ricoeur, P. Larre, C. Panikkar, R. Kagame, A. Lloyd, G. Neher, A. Pattaro,G. Gardet, L.Gouveritch, A. (1975). Las culturas y el tiempo. Estudios resumidos para UNESCO. Vozes. Editorila de la Universidad de Sao Paulo.

Ricoeur, P. (1995). Tiempo y narración (Vol. I). Madrid - España: Siglo XXI Editores.

Rivarola, J. (1994). La no existencia física del tiempo: revelación de un enigma milenario. Imprenta salesiana.

Rodriguez, L \& Romero, A. (1999). La construcción de la historicidad de las ciencias y la transformación de las práctica pedagógicas. Fisica y Cultura: Cuadernos sobre Historia y Enseñanza de las ciencias (6).

Saavedra, I. (1999). El tiempo en la física. Anales de la Universidad de Chile .

Toledo, U. (1998). La epistemología según Feyerabend. Cinta de Moebio (004). 